\title{
"The PLCP gene family of grapevine (Vitis vinifera $L$.): characterization and differential expression in response to Plasmopara Viticola"
}

\author{
Jun Kang ${ }^{\dagger}$, Peijie Gong ${ }^{* \dagger}$, Mengqing Ge, Ehsan Sadeghnezhad, Zhongjie Liu, Mengwei Zhang, \\ Lingfei Shangguan and Jinggui Fang*
}

\begin{abstract}
Background: Papain-like cysteine proteases (PLCPs), a large group of cysteine proteases, are structurally related to papain. The members belonging to PLCPs family contribute to plant immunity, senescence, and defense responses in plants. The PLCP gene family has been identified in Arabidopsis, rice, soybean, and cotton. However, no systematic analysis of PLCP genes has been undertaken in grapevine. Since Plasmopara viticola as a destructive pathogen could affect immunity of grapes in the field, we considered that the members belonged to PLCPs family could play a crucial role in defensive mechanisms or programmed cell death. We aimed to evaluate the role of PLCPs in 2 different varieties of grapevines and compared the changes of their expressions with the transcriptional data in response to $P$. viticola.
\end{abstract}

Results: In this study, 23 grapevine PLCP (VVPLCP) genes were identified by comprehensive bioinformatics analysis. Subsequently, the chromosomal localizations, gene structure, conserved domains, phylogenetic relationship, gene duplication, and cis-acting elements were analyzed. Numerous cis-acting elements related to plant development, hormone, and stress responses were identified in the promoter of the VVPLCP genes. Phylogenetic analysis grouped the VVPLCP genes into nine subgroups. The transcription of VVPLCP in different inoculation time points and varieties indicated that $V V P L C P$ may have vital functions in grapevine defense against Plasmopara viticola. According to transcriptome data and qPCR analysis, we observed the increasing expression levels of $V_{V} R D 21-1$ at $72 \mathrm{~h}$ after inoculation in resistant variety, inferring that it was related to grape downy mildew resistance. Meanwhile, 3 genes including VVXBCP1, VVSAG12-1, and VVALP1 showed higher expression at 24h after pathogen inoculation in the susceptible variety and might be related to the downy mildew phenotype. We nominated these four genes to function during hypersensitive response (HR) process, inferring that these genes could be associated with downy mildew resistance in grapes.

Conclusions: Our results provide the reference for functional studies of PLCP gene family, and highlight its functions in grapevine defense against $P$. viticola. The results help us to better understand the complexity of the PLCP gene family in plant immunity and provide valuable information for future functional characterization of specific genes in grapevine.

Keywords: Grapevine, Papain-like cysteine proteases, Phylogenetic analysis, Gene expression, Plasmopara viticola

\footnotetext{
*Correspondence: gongpeijie@njau.edu.cn; fanggg@njau.edu.cn

† Jun Kang and Peijie Gong contributed equally to this work.

Department of Horticulture, Nanjing Agricultural University,

Nanjing 210095, Jiangsu Province, China
} permitted by statutory regulation or exceeds the permitted use, you will need to obtain permission directly from the copyright holder. To view a copy of this licence, visit http://creativecommons.org/licenses/by/4.0/. The Creative Commons Public Domain Dedication waiver (http://creativecommons.org/publicdomain/zero/1.0/) applies to the data made available in this article, unless otherwise stated in a credit line to the data. 


\section{Background}

Papain-like cysteine proteases (PLCPs) are a class of proteolytic enzymes, with a catalytic cysteine as a nucleophile during proteolysis [1]. PLCP belongs to the family $\mathrm{C} 1 \mathrm{~A}$ of clan $\mathrm{CA}$, which are widely found in viruses, bacteria, yeast, protozoa, plants, and animals [2, 3]. PLCPs are structurally characterized by a typical papain-like fold domain: an $\alpha$-helix and $\beta$-sheet domains [2]. Both domains are linked to each other, and the catalytic triad Cys-His-Asn is formed at the two-domain interface [4]. PLCPs are synthesized as preproproteases, which contain a signal peptide, an auto-inhibitory pro-domain, and a mature protease domain [5]. After cleaving off an inhibitory pro-domain, PLCPs become active through selfprocessing or with the aid of processing enzymes [6]. The optimum pH for catalytic activity of PLCPs is $5.0-8.0$ and the range of molecular masses for most PLCPs changes from 20 to $35 \mathrm{kDa}$, and a few are $50-75 \mathrm{kDa}$ [7]. In the first classification of 138 plant PLCPs, eight subfamilies were identified based on their structural characteristics [8]. Later, 723 plant PLCPs were determined and grouped into nine classes according to domain architecture and their homology [9, 10]. In Arabidopsis, 31 PLCPs were classified into nine subfamilies including; CTB-like subfamily (i.e., B-like), ALP-like subfamily (i.e., H-like), RD19-like subfamily (i.e., F-like), SAG12-like subfamily (i.e., L-like), THI-like subfamily (i.e., L-like), XBCP-like subfamily (i.e., L-like), XCP-like subfamily (i.e., L-like), CEP-like subfamily (i.e., L-like), and RD21-like subfamily (i.e., L-like) [11]. The ALP-like subfamily members usually contain a vacuolar-targeting motif NPIR at the $\mathrm{N}$-terminus of the protease precursor. The CEP-like subfamily members as a unique group of papain-type cysteine endopeptidases are characterized by a $\mathrm{C}$-terminal KDEL motif for retention in the endoplasmic reticulum (ER). Most members in the RD21-like and XBCP-like subfamilies carry a C-terminal extension with a Pro-rich domain followed by a granulin-like domain [12].

PLCPs are also involved in diverse biological processes including senescence [13], growth development, and defense responses [14]. As an essential part of the proteolytic machinery, PLCPs are responsible for the degradation of intracellular proteins and are key enzymes in the regulation of programmed cell death (PCD). PCD is genetically programmed and led to the cell suicide process and the removal of damaged cells. Furthermore, it is a tightly regulated biological process that functions in many aspects of plant development and the responses to biotic and abiotic stresses. Increased activity of PLCPs was observed in developing and germinating seeds [15], fruits [16], and senescing organs [17]. In Arabidopsis, AtSAG12 exhibits a strictly senescence-associated expression pattern in leaves, which is widely used as a molecular marker for the study of leaf senescence. Furthermore, AtRD21 and AtRD19 were applied as marker genes for early responsive to dehydration under drought and salt stresses [18, 19]. Meanwhile, PLCPs play a critical role under herbivore attack and activate the resistance mechanisms in plants. For example, Papain, one of the PLCPs in latex exuding from wounds, could be involved in the protection mechanisms induced by herbivorous insects in papaya [20]. Similarly, a 33-kDa PLCPs in maize damaged the digestive systems related to caterpillars and induced resistance to them [21].

Grape (Vitis L.) is one of the most important economic fruits and is widely cultivated all over the world for the production of wine, juice, fresh food, and dried fruit. Among various biological stresses, the downy mildew of grape causes by a very destructive pathogen, which brings huge economic losses to viticulture worldwide [22]. Plasmopara viticola is the causative agent of grape downy mildew and is considered to be the most destructive grapevine disease in a relatively warm and humid climate. Almost all Eurasian grapevines are susceptible to downy mildew, and the symptoms of the disease are similar, which the main infections damage the leaves, young shoots, and young fruits [22, 23]. The activity of PLCPs is required to trigger plant immune responses and fulfill effective defense against pathogen infection [24, 25]. Meanwhile, PLCPs are often targeted by pathogen-derived effectors to suppress plant immune responses [26]. Therefore, the continuous coevolutionary arms race between pathogens and their hosts might have driven a more rapid evolution of plant PLCPs compared to the rest of plant genomes [27].

Although the PLCP family has been identified in Arabidopsis [19], cotton [28], rice [14], and soybean [29], yet no comprehensive study of PLCP genes has been reported in grapevine. With the release of the grapevine genome, we obtained the necessary information for bioinformatics analyses of the $V v P L C P$ family and systematically investigated the putative functions of $P L C P$ genes in grapevine. In this study, 23 non-redundant members of the $V v P L C P$ gene family were characterized in resistant and susceptible grapevine varieties, respectively. Subsequently, the detailed gene structure, phylogenetic relationship, GO pathway and expression profile under P. viticola infection were investigated. And hypersensitive response (HR) was observed in resistant grapevine varieties. Our results provide exact information on the $V v P L C P$ family for further functional characterization of $P L C P$ genes in downy mildew of grape. 


\section{Results}

Identification of $V v P L C P$ gene family in grapevine

To identify and obtain the PLCP genes in the grapevine genome, the Arabidopsis PLCP proteins were used as a query to search against the local grapevine genome database using TBtools software. Then, the hidden Markov model (HMM) profile of the Peptidase_C1 domain (PF00112) was employed to perform a global search of the grapevine genome. After analyzing the conserved domain and removing the redundant sequences, a total of 23 putative $V v P L C P$ genes were identified in grapevine. For the sake of nomenclature and consistency, these $V v P L C P$ genes depending on their homology to the Arabidopsis PLCP members were named $V v R D 21-1 \sim 3$, $V \nu C E P 1 \sim 2, \quad V v X C P 1 \sim 2, \quad V v X B C P 1 \sim 2, V v S A G 12-1 \sim 6$, $V v R D 19-1 \sim 5, V v A L P 1, V v C T B 1$ and VvTHI1 (Additional file 1 Table $\mathrm{S} 1$ ).

\section{Phylogenetic analysis of PLCP in Arabidopsis thaliana, Oryza sativa, Glycine max, and Vitis vinifera}

To investigate the phylogenetic relationship of grapevine $P L C P$ with those of other plants, we conducted a full-length peptide sequence alignment among $23 \mathrm{VvPL}$ CPs (Vitis vinifera), 31 AtPLCPs (A. thaliana), 45 OsPLCPs (O. sativa), and $97 \mathrm{GmPLCPs}$ (Glycine max) using the MEGA software. The PLCPs from these four different species were distributed in nine distinct subfamilies (Fig. 1), which is consistent with a previous study [4]. According to the previous report [4], we named nine subfamilies $R D 21$ (responsive to desiccation 21), CEP (cysteine endopeptidase), $X C P($ xylem cysteine peptidase), $X B C P$ (xylem bark cysteine peptidase), THI(THIlikesubfamily), $S A G 12$ (senescence-associated gene 12), $R D 19$ (responsive to desiccation 19), $A L P$ (aleurain-like protease), and $C T B$ (cathepsin B-like). Phylogenetic tree analysis revealed that the members belonged to $P L C P S$ are not evenly distributed among the nine subfamilies. The subfamily $S A G 12$ is the largest subfamily with 6 members, while three subfamilies including $T H I$, $A L P$, and $C T B$ contain only 1 member. The subfamilies $R D 21$ and $R D 19$ contain 3 and 5 members, respectively. While, the subfamilies $X C P, X B C P$, and $C E P$ contain 2 PLCPs, respectively. These results suggest that subfamily SAG12 shows large conservation and might share similar functions to its abundance. The detailed information of $V v P L C P$ was listed in Additional file 2 Table S2, including gene name, protein length, chromosome location, molecular weight, theoretical isoelectric point, and aliphatic index. The $23 V v P L C P$ proteins had diverse molecular lengths and weights, ranging from 186 ( VvRD19-4) to 501 (VvSAG12-4) in amino acid length. $V v R D 19-4$ showed the lowest value of the molecular weight $(20.67 \mathrm{kDa})$, while the highest molecular weight
$(55.94 \mathrm{kDa})$ was observed in $V v S A G 12-4$. Theoretical isoelectric points of these $V v P L C P$ proteins varied from 4.87 $(V v S A G 12-4)$ to 7.62 ( $V v R D 19-4)$ and the value of the aliphatic index was ranged from $19.52(V v X C P 2)$ to 55.51 ( $V v S A G 12-4)$. In addition, according to subcellular prediction analysis by WoLF PSORT website, we found the 8 and $9 V v P L C P$ proteins that were located on the chloroplast and the extracellular, respectively, although, a few proteins were located in other subcellular compartments, such as vacuole, chloroplast, and endoplasmic reticulum (ER) lumen (Additional file 2 Table S2).

\section{Gene structure and distribution of conserved motif of PLCPs}

Multiple sequence alignment was employed to explore sequence features and MEME website analysis was performed to predict distinct motifs. The length of $V v P L C P$ proteins varied widely from 186 to 501 amino acids. Among them, $21 V v P L C P s$ were found to contain one Inhibitor_I29 domain and a conserved Peptidase_C1 domain. One member consisted of one Peptidase_C1 domain but no Inhibitor_I29 domain. One $V v P L C P$ contained only two Peptidase_C1 domains, and four with not only one Inhibitor_I29 and Peptidase_C1 domain but also one granulin-like domain (Additional file 3 Fig. S1). A total of 15 motifs named motifs $1-15$, were finally identified (Fig. 2 A, B and Additional file 3 Fig. S1). The type, number, order, and motif location in $V v P L C P S$ were similar within each subfamily but differed from each other. Firstly, Motifs 5, 6, and 11 were characterized as the Inhibitor_I29 domain (PF08246), they were present in the other 7 subfamilies except for the CTB subfamily and SAG12 subfamily. Motif 15 is specific to subfamily SAG12. Secondly, motifs 1, 2, 3, 4, 5, 7, 8, 9, 10, and 12 are characterized as the Peptidase_C1 domain(PF00112). Motif 1, 2, and 4 have Cys, His, and Asn in catalytic sites, respectively. Catalytic triad (Cys-His-Asn) is conserved in Vitis vinifera except for $V v S A G 12-6$ from subfamily $S A G 12$, in which $H i$ s are substituted with $A s n$ in the active site. Thirdly, motif 13, which is included in subfamily $X B C P$ and two members in the subfamily $R D 21$, are characterized as the granulin-like domain (PF00396).

The gene structure of PLCPS was also analyzed, and the results showed that these genes contain more than two exons, range from two to ten (Fig. 2 C). Generally, a certain subfamily has a highly conserved exon-intron structure but different subfamilies have distinct structures. Subfamily SAG12 features two or three exons. Among them, $S A G 12-3$ and $S A G 12-6$ have two introns. Subfamilies CEP, XCP, and RD19 features four exons and three introns, while subfamily $X B C P$ features five or six exons and four or five introns. Subfamily RD21 usually features four or five exons and three or four introns. Subfamily 


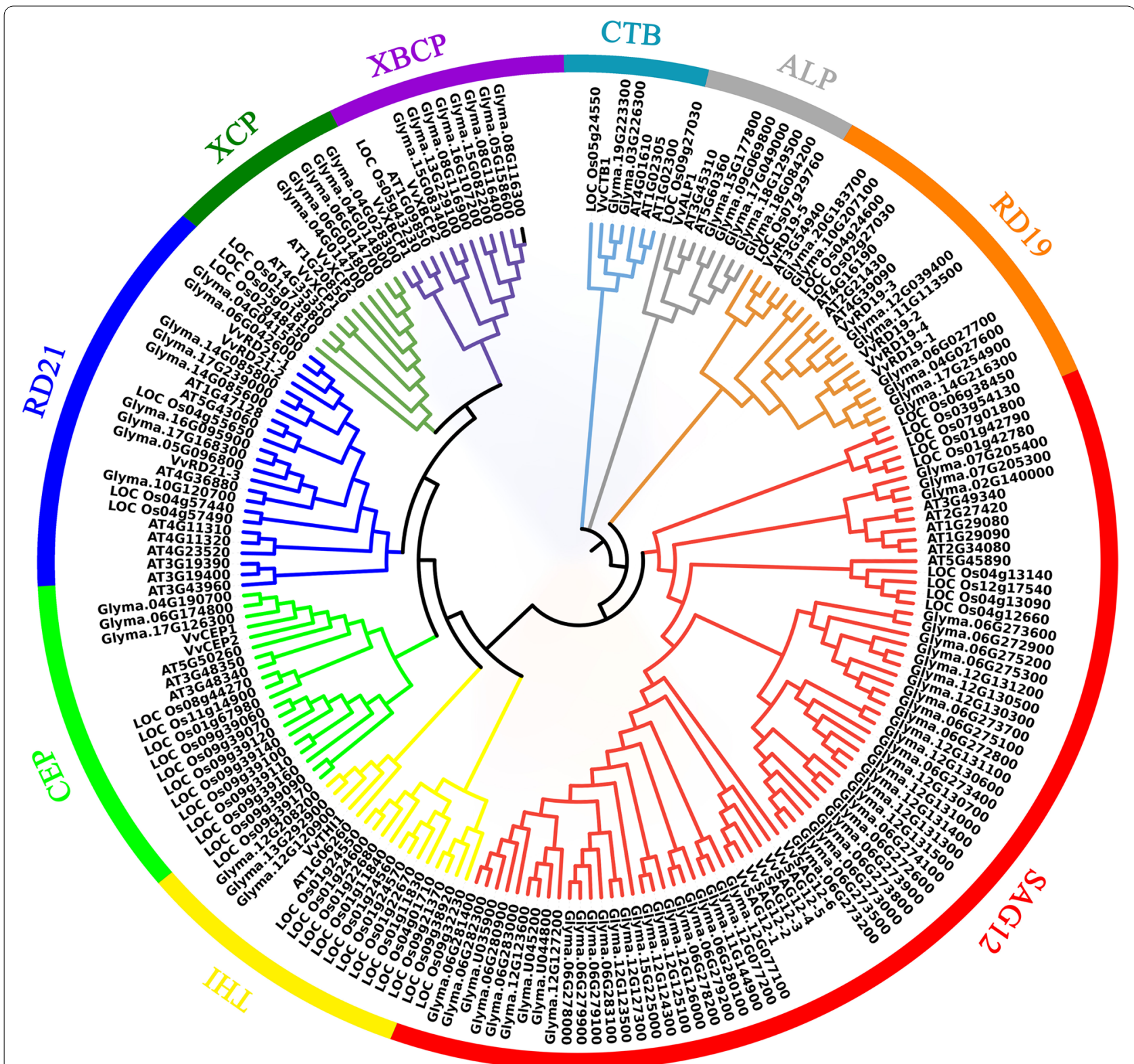

Fig. 1 Phylogenetic relationships of V. vinifera VVPLCP proteins with A. thalinana, O. sativa, and Glycine max. The phylogenetic tree was drawn by using the maximum likelihood (ML) method with 1000 bootstrap replicates in MEGA sofeware

$A L P$ features seven exons, while Subfamily $C T B$ and $T H I$ feature ten and two exons, respectively. $V v C T B 1$ contained the highest number of exons (10), VvSAG12-1, $V v S A G 12-2, V v S A G 12-6$, and VvTHI1 only had two exons among the $V v P L C P s$. Overall, the structures and motifs supported the results of phylogenetic analysis.

\section{Gene duplication analysis and chromosomal distribution of $V v P L C P s$}

To understand the genomic distribution and gene duplication of $V v P L C P$ genes, $23 V v P L C P$ genes were distributed unevenly throughout the 10 out of the 19 chromosomes according to the current grapevine genome (Fig. 3). Among them, chromosomes 12 and 18 had the highest number of VvPLCP genes (five), while chromosomes $1,4,11,13$, and 17 had only one $V v P L C P$ gene. Three $V v P L C P$ genes were located on each of chromosomes 3 and 10 and two $V v P L C P$ genes were distributed on chromosome 7. Tandem duplication and segmental duplications occurred frequently in gene families' evolution and expansion. Tandem duplication usually causes the development of the gene 

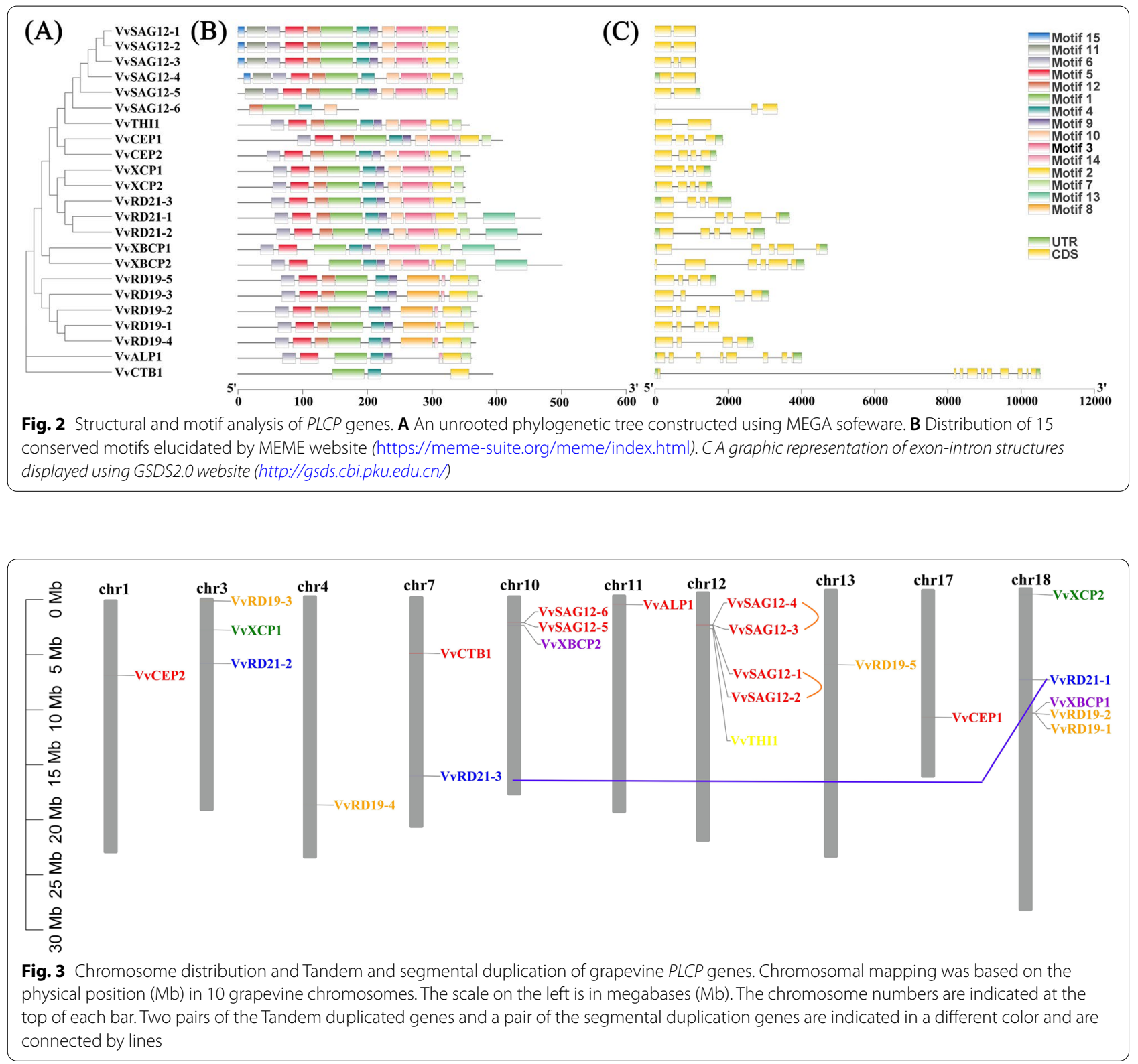

clusters, although segmental duplication might scatter the members belonged to a gene family [30]. There are two tandem duplication clusters (VvSAG12-3/ VvSAG12-4 and VvSAG12-1/VvSAG12-2) in VvPLCP gene family, which were identified on grapevine chromosome 12(Fig. 3). Then, a pair of duplicated segments $(V v R D 21-1 / V v R D 21-3)$ in $V v P L C P$ gene family were identified within the grapevine genome. Our results suggest that tandem duplication events may be more important than segmental duplication in the expansion of the $V v P L C P$ gene family in grapevine. Furthermore, we also calculated the value of $\mathrm{Ka} / \mathrm{Ks}$ of duplication genes pairs, which could be used as an indicator for the selection pressure of a gene during evolution. The results showed that all the $\mathrm{Ka} / \mathrm{Ks}$ values were less than 1 , indicating that the $V v P L C P$ genes primarily evolved under the influence of purifying selection (Additional file 4 Table S3). 


\section{Identification of cis-elements in the promoters of $V v P L C P$ genes}

To better understand the transcriptional regulation and the gene function of $V v P L C P s$, the cis-elements in the promoter regions of the $V v P L C P(2 \mathrm{~kb}$ upstream of the translation start site in the genomic DNA sequence) were used to search in PlantCARE database and visualized by TBtools software (Additional file 5 Table S4 and Additional file 6 Fig. S2). As expected, CAAT-box and TATA-box, the conventional promoter elements, were found in all the $V v P L C P$ promoters. A series of cis-elements involved in plant growth and development, phytohormone responses, and stress responses were identified. The CAT-box involved in meristem expression was identified in the promoters of $3 V v P L C P$ genes. The binding site of AT-rich DNA binding protein (ATBP-1) was found in $6 V v P L C P$ genes. Additionally, the seed-specific regulation element (RY element) and endosperm expression regulation element (GCN4_motif) were also found in the promoters of the $V v P L C P$ genes. Among the cis-acting elements involved in hormone responses, the abscisic acid responsive element (ABRE), the MeJA responsive element (CGTCA-motif and TGACG-motif) and gibberellin-responsive element (GARE-motif, P-box and TATC-box) were found in the promoters of 22, 17, and 13 $V v P L C P$ genes, respectively. Salicylic acid (SA) responsive element (TCA-element) and auxin-responsive element (AuxRR-core, AuxRE, and TGA-element) were also observed in 11 and $10 \mathrm{VvPLCP}$ genes (Additional file 5 Table S4). Among stress-related responses elements, ARE, which was the most abundant element and involved in an aerobic induction, was observed in promoter regions of $V v P L C P$ genes. In addition, some stress related cis-acting elements (low-temperature and wound) were also found in the promoter regions of the $V v P L C P$ genes. There were some MYB binding responses elements, such as MBS and MBSI, in grapevine PLCP. Meanwhile, a number of light response elements were also found in the promoter regions of $V v P L C P$, including Box 4, G-Box, G-box, GATA-motif, GT1-motif, I-box, MRE, TCCCmotif and TCT-motif.

\section{$P$. viticola BS-4-MW induced HR-like necrotic spots in Vitis rootstocks 'Kober 5BB' as resistant cultivar}

$V$. vinifera $c v$. 'Zitian Seedless' and Vitis rootstocks 'Kober 5BB' were classified as susceptible and resistant genotypes, respectively. They were selected for a more detailed temporal analysis of the necrotic lesion formation. After $P$. viticola strains infection, the two Vitis cultivars showed different infection symptoms: HR-like necrotic spots could be observed on 'Kober 5BB', meanwhile downy mildew sporangia were formed on $c v$. 'Zitian Seedless' leaves (Fig. 4 A, B). These regions of local cell death could be observed on leaf discs at 7 days post-infection, although, were never seen in the susceptible grape cv. 'Zitian Seedless'. The density of the necrotic spots increased more in time after inoculation (Fig. 4 C, D).

\section{GO and expression patterns analysis of VvPLCP genes in grapevine infected with $P$. viticola}

Gene ontology (GO) of the grapevine PLCP was investigated based on the putative assignment of $10 \mathrm{GO}$ terms using the data in grapevine Genome Database. These GO function terms were divided into three categories: biological process, cellular components, and molecular function (Additional file 7 Fig. S3), and the detailed gene ID information of them was shown in Additional file 8 Table S5. In biological processes, 'proteolysis involved in cellular protein catabolic process(GO:0051603)', and 'cytokinesis and cellular protein catabolic process(GO:0044257)' were the most enriched GO terms. Similarly, 'cysteine-type endopeptidase activity(GO:0004197)', 'cysteine-type peptidase activity(GO:0008234)', and 'endopeptidase activity(GO:0004175)' were enriched in terms of molecular function. Other GO terms, such as, 'lysosome(GO:0005764)', 'lytic vacuole(GO:0000323)', 'extracellular space(GO:0005615)', 'extracellular region part(GO:0044421)', and 'vacuole(GO:0005773)' were the most common GO terms in the cellular component.

In order to investigate the putative roles of the $V v P L C P$ genes in grapevine resistance, the leaves-specific expression patterns of $V v P L C P S$ were analyzed according to transcriptome data in two cultivars including $V$. vinifera $c v$. 'Zitian Seedless' and Vitis rootstocks 'Kober 5BB' when infected with $P$. viticola (Additional file 9 Table S6). As shown in Fig. 5, in the $V$. vinifera cv. 'Zitian Seedless' as susceptible cultivar, five $V v P L C P s$ including $V v R D 19-$ 5, $V v R D 19-3, V v R D 21-2, V v X B C P 1$, and $V v C E P 1$ genes were ubiquitously high expressed, while six $V v P L C P$ including $V v R D 19-1, V v R D 19-4, V v C T B 1, V v X B C P 2$, $V \nu A L P 1$, and $V \nu C E P 2$ genes down-regulated at $0 \mathrm{~h}$. At 24 and $48 \mathrm{~h}$ after $P$. viticola infection of susceptible grapevine, three genes $(V v X B C P 2, V v R D 19-3$, and $V v R D 19-$ 2) and eight $V v P L C P$ genes ( $V v R D 19-2, V v R D 21-1$, $V v R D 21-3, V v X B C P 1, V v X B C P 2, V v X C P 1, V v X C P 2$ and $V v A L P 1)$ were up-regulated but two $V v P L C P$ ( $V v T H I 1$ and $V v C T B 1)$ genes indicated a decrease in their expression level at 24h, although some VvPLCPs ( VvRD19-1, $V v R D 19-5, V v C E P 1$ and $V v C T B 1)$ were almost undetectable at $72 \mathrm{~h}$ after downy mildew infection.

In Kober $5 \mathrm{BB}$ as resistant cultivar, five genes including VvSAG12-5, VvTHI1, VvCTB1, VvALP1, and VvRD19-4 displayed the high level of expression but $V v X B C P 1$ and $V v R D 21-3$ expressed at a very low level at $0 \mathrm{~h}$. After $24 \mathrm{~h}$ of $P$. viticola infection in resistant cultivar, two $V v P L C P$ $(V v C E P 2$ and $V v R D 19-1)$ genes were ubiquitously 


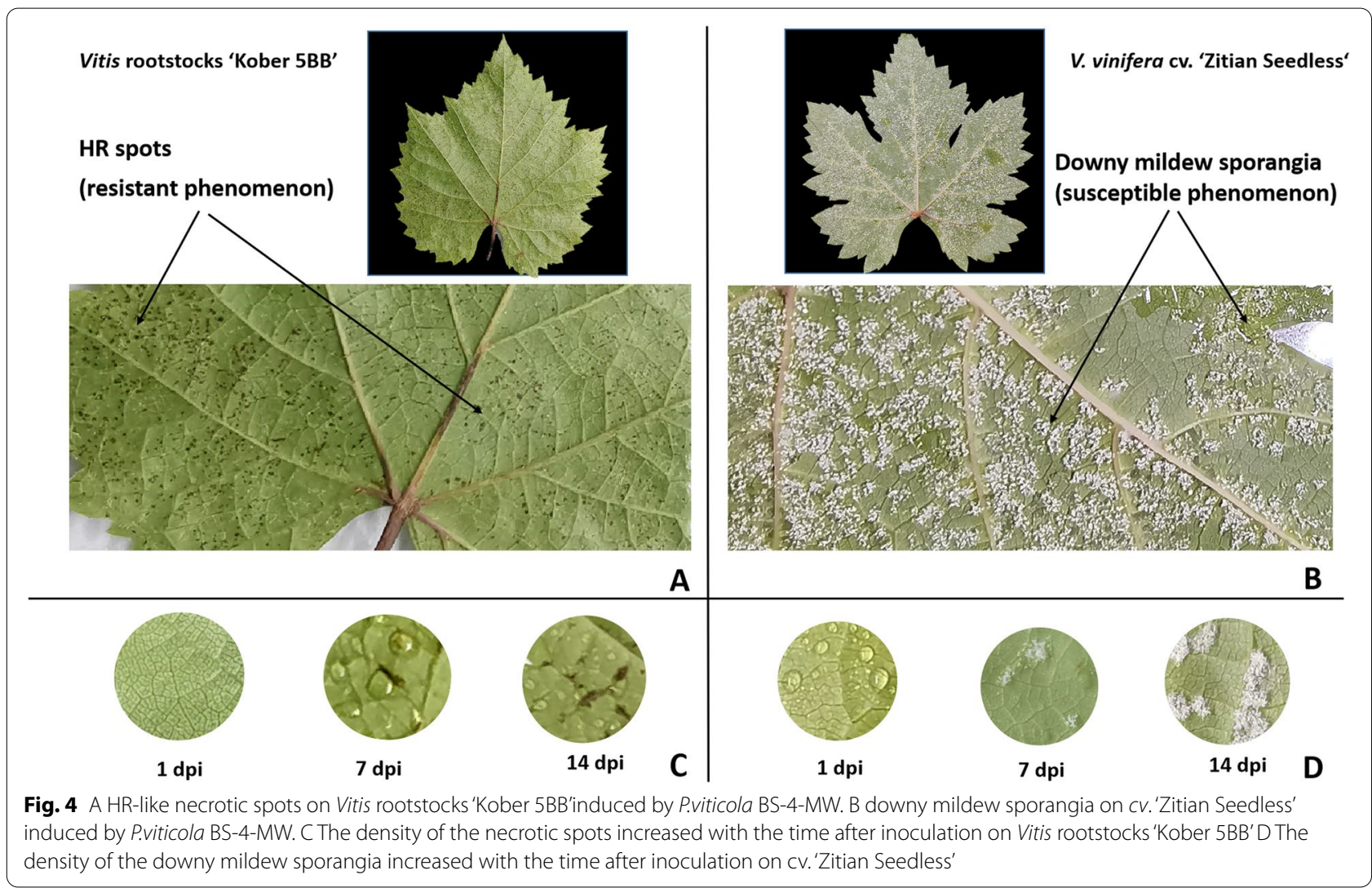

high expressed and five $V v P L C P(V v X B C P 2, V v R D 21-$ 1, $V v R D 19-3, V v R D 19-4$, and $V v A L P 1)$ genes were expressed at a very low level. Two $V v P L C P$ ( $V v S A G 12-6$ and $V v R D 19-1)$ genes were high expressed, whereas $V v R D 21-2$ and $V v S A G 12-5$ were expressed at a very low level at $72 \mathrm{~h}$ after infection with $P$. viticola in Kober $5 \mathrm{BB}$ cultivar. On the whole, the gene expression was specific at different treatment time points, except for four VvPLCP (VvSAG12-1, VvSAG12-2, VvSAG12-3, and $V v S A G 12-4)$ genes, which remained unchanged in the $V$. vinifera cv. 'Zitian Seedless' and Vitis rootstocks 'Kober 5BB'.

\section{qPCR validation of $V v P L C P$ genes in $P$. viticola infection of grapevine}

Many studies have shown that PLCP gene family plays an important role in growth and development. In order to further verify the changes of PLCPS expression in resistant and susceptible grapes under downy mildew (DM) disease caused by $P$. viticola, the leaves-specific expression patterns of $V v P L C P s$ were analyzed in the $V$. vinifera $c v$. 'Zitian Seedless' and Vitis rootstocks 'Kober 5BB' at seven different times such as $0,12,24,48,72,96$, and $168 \mathrm{~h}$ by qPCR analysis. As shown in Fig. 6-1 and Fig. 62, $23 P L C P$ genes were expressed in the $V$. vinifera $c v$.
'Zitian Seedless' and Vitis rootstocks 'Kober 5BB'. In Vitis rootstocks 'Kober 5BB', 7 PLCP genes were up-regulated after $168 \mathrm{~h}$ of inoculation, 5 PLCP genes were down-regulated, and 11 PLCP genes showed no significant changes after $0 \mathrm{~h}$. In $V$. vinifera $c v$. 'Zitian Seedless', 14 and 3 PLCP genes were up- and down-regulated at $168 \mathrm{~h}$ after inoculation, respectively, while 6 PCLP genes showed no significant changes within one week before and after inoculation. In these two grapevine varieties, the prominently expressed $P L C P$ genes are basically from the $R D 21$, $C E P$ and $S A G 12$ subfamilies. Significant expression of members belonged to $X B C P$ and $R D 19$ subfamilies was also found in $V$. vinifera $c v$. 'Zitian Seedless' and there was almost no change in Vitis rootstocks 'Kober 5BB' (Fig. 6-1 and Fig. 6-2). In the three time periods of 0, 24, and $72 \mathrm{~h}$ after pathogen exposure, we observed the different patterns of genes expression in both susceptible and resistant grapevine cultivars. For example, 5 PLCP genes in Vitis rootstocks 'Kober 5BB' were down-regulated at $24 \mathrm{~h}$ and then up-regulated at $72 \mathrm{~h}$, while 8 PLCP genes were up-regulated at $24 \mathrm{~h}$ and then down-regulated at $72 \mathrm{~h}$. Meanwhile, one PLCP genes continued to be downregulated and $5 P L C P$ gene continued to be up-regulated, although 4 PLCP genes had no significant changes. Among them, $V v R D 21-1$ showed higher expression at 


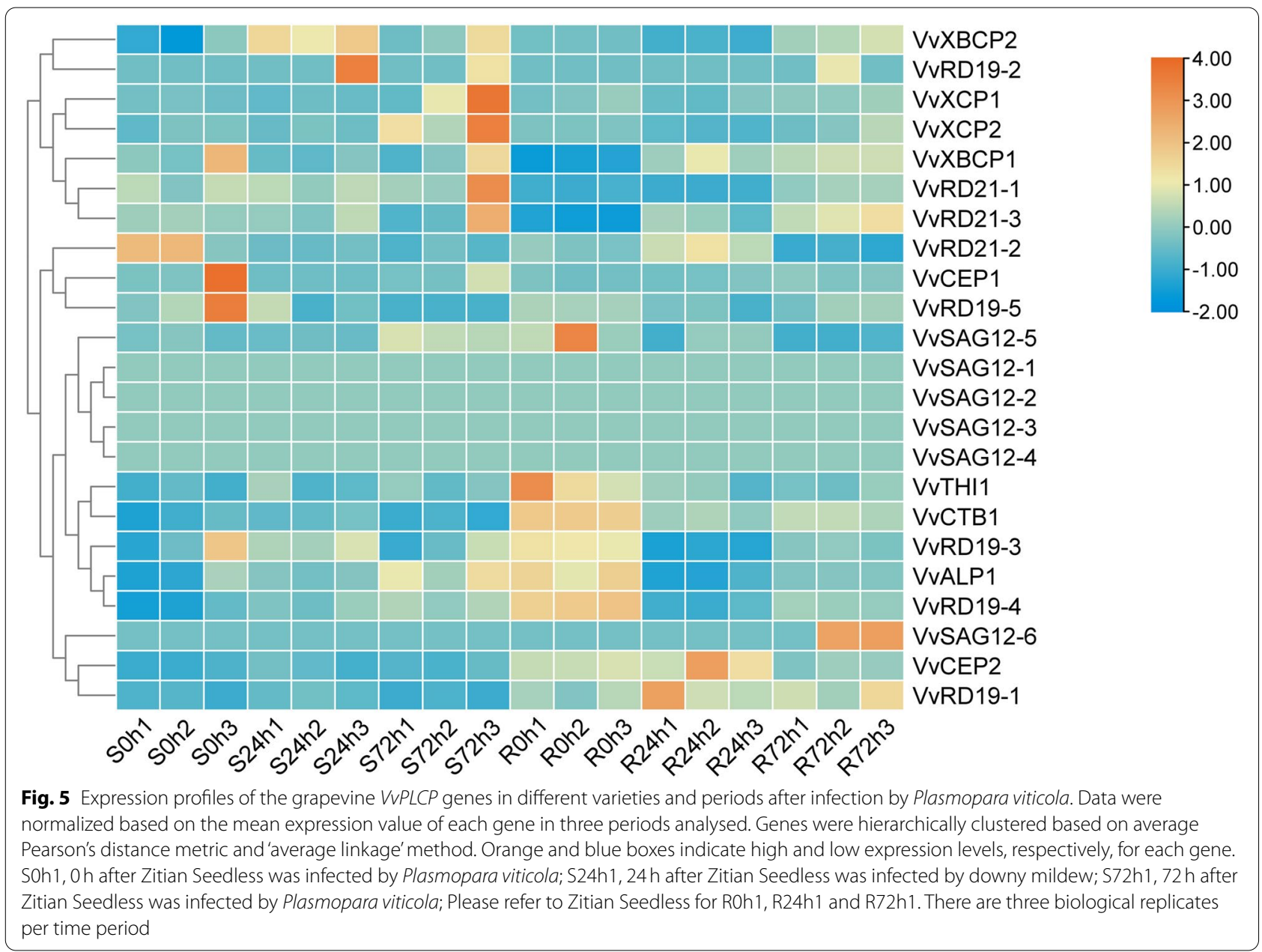

$72 \mathrm{~h}$ after inoculation. Similarly, in $V$. vinifera $c v$. 'Zitian Seedless', 12 PLCP genes were up-regulated at $24 \mathrm{~h}$ and then down-regulated at $72 \mathrm{~h}, 1 P L C P$ genes were downregulated at $24 \mathrm{~h}$ and then up-regulated at $72 \mathrm{~h}, 2 P L C P$ genes continued to be down-regulated, and one $P L C P$ gene had no significant change. Among them, 2 genes including $V v X B C P 1, \quad V v A L P 1$ and $V v S A G 12-1$ showed much higher expression at $24 \mathrm{~h}$ (Fig. 6-1 and Fig. 6-2). These gene expression trends at 0,24 , and $72 \mathrm{~h}$ are basically consistent with the transcriptome analysis data. The expression changes of these genes imply that they play an important role in response to $P$. viticola.

\section{Discussion}

As one of the most abundant family of cysteine proteases in plants, PLCPs are proteolytic enzymes that are involved in a broad range of biological processes such as senescence, PCD, pollen development, fruit ripening, and seed germination [13]. Genome-wide scan of PLCPs has been systematically carried out in Arabidopsis (Arabidopsis thaliana) [4], rice (Oryza sativa L.) [14], castor bean (Ricinus communis) [31], physic nut (Jatropha curcas) [31], papaya (Carica papaya L.) [32], Fig (Ficus carica L.) [33] and rubber (Hevea brasiliensis) [34]. In this study, we performed a genome-wide analysis of the $P L C P$ genes in two varieties of grapevine including resistant and susceptible cultivars. Although the number of PLCP genes is less than that of the other plants such as 78 for cotton [28], 31 for Arabidopsis [30], 33 for rice [14], 97 for soybean [31], and 31 for Fig [33].. The difference might be due to the variable state of paralogous genes in these genomes. For example, only 1 paralogous gene pairs were found in grapevine, while 24 paralogous and 19 paralogous gene pairs from segmental duplication events were identified in cotton and soybean, respectively.

In addition, there are two tandem duplication clusters: (VvSAG12-3/VvSAG12-4 and VvSAG12-1/VvSAG12-2) in $V v P L C P$ gene family, which was identified on grapevine chromosome 12 while only a pair of duplicated segments (VvRD21-1/VvRD21-3) in VvPLCP gene family. It reveals that tandem duplication events are more likely than segmental duplication events in grapevine. This 


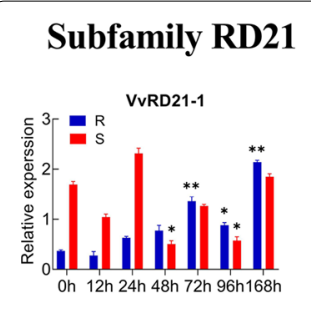

Subfamily XCP

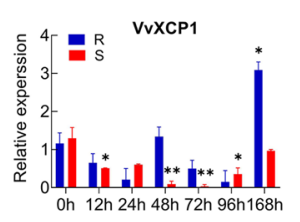

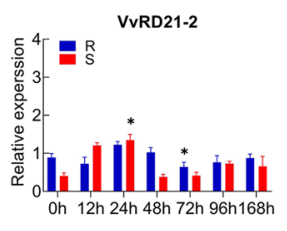

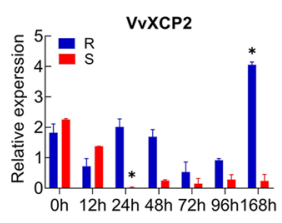

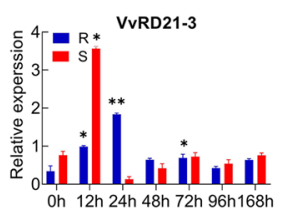

Subfamily XBCP

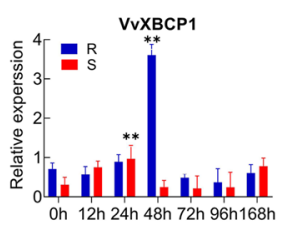

\section{Subfamily CEP}
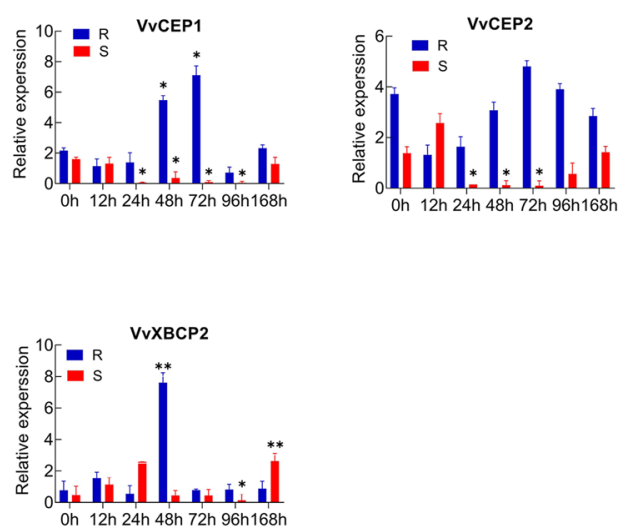

\section{Subfamily RD19}
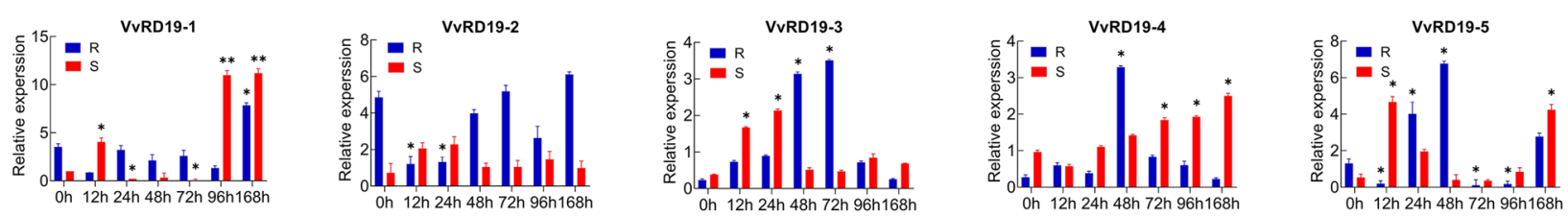

Subfamily SAG12
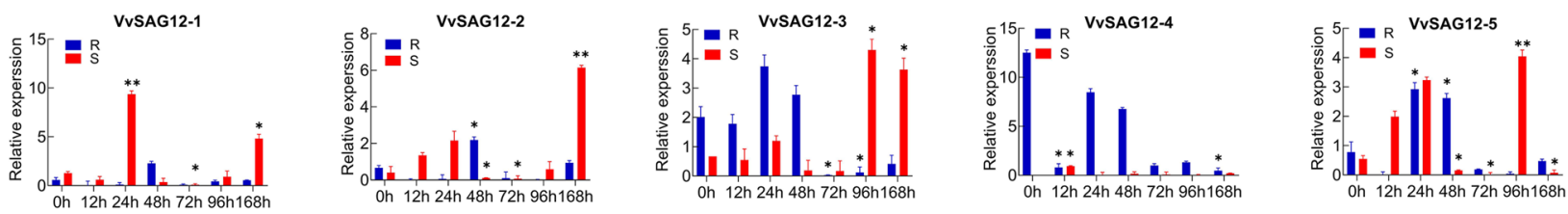

Subfamily THI
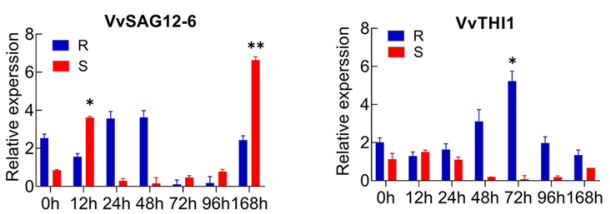

Subfamily CTB

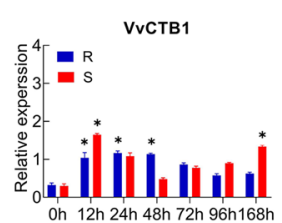

\section{Subfamily ALP}

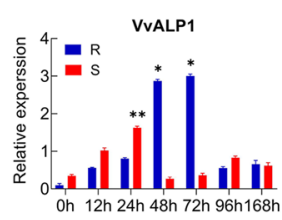

Fig. 6 qPCR analysis of 23 VVPLCPs in in two varieties after infection by Plasmopara viticola. The expression level in $0 \mathrm{~h}$ was used as a starting point control to calculate the relative expression levels of other samples. Asterisks indicate that the corresponding genes were distinctly up- or down-regulated following different inoculation time points by t-test $\left.{ }^{*} P<0.05,{ }^{*} P<0.01\right)$. The mean and SD values were derived from three biological and three technical repetitions. The MIQE guidelines were followed for performing the qPCR experiments of $P L C P$ genes. $S C V$. 'Zitian Seedless'. R Vitis rootstocks 'Kober 5BB'. Note, see Fig. 6-1 and Fig. 6-2

conclusion was supported by the previous study [32]. Furthermore, these PLCP genes were grouped into nine subfamilies according to their phylogenetic clades and structure features, which were also corresponding to a previous report [8]. Meanwhile, genes number in each subfamily is out of proportion among some plant species. According to the past PLCPs identification research, we infer that the significant variations of gene number in each subfamily may be caused by the issues of whole genome duplication, tandem duplication, and large-scale segmental duplication in various plant evolution periods.
Furthermore, the value of $\mathrm{Ka} / \mathrm{Ks}$ of segmental gene pairs was also calculated. Generally, Ka/Ks ratio greater than 1 , equal to 1 , and less than 1 represent positive selection, neutral selection, and negative selection, respectively. Remarkably, the $\mathrm{Ka} / \mathrm{Ks}$ ratios of all grapevine gene pairs were less than 1 , indicating that these gene pairs have been experiencing a markedly purifying selection during their evolution to prevent the spread of deleterious mutations.

In natural environment, plants are attacked by a diverse array of pathogens and pests, including bacteria, fungi, 
oomycetes, nematodes, insects, and microbes. Many studies have highlighted the importance of PLCP in defense against plant pathogen $[25,35]$. In most cases, a lack of $P L C P$ expression leads to alterations of pathogen resistance because PLCP mutations are more susceptible to pathogen infection [25]. For example, Arabidopsis null mutants for the $R D 21$ subfamily members are more susceptible to the necrotrophic fungal pathogen Botrytis cinerea, although these lines were more resistant for the same pathogen in detached leaf assays before $R D 21$ is missing [36]. Interestingly, we found that the VvRD21-1 might play an important role in grape disease resistance. Previous studies [37] showed that the knocking out or silencing $R D 21$ subfamily members makes plants more susceptible to necrotrophic fungal and oomycete biotrophic pathogens. Similarly, in cotton, orthologs GhRD21-7 of VvRD21-1 were also found to have higher resistance to $V$. dahliae [28].

According to biotic stress of variant plants, PLCPs are also regarded as key regulators of SA-dependent defense signaling. Previous studies [35] showed that SA treatment could strongly induce PLCP protein activity in maize leaves, and the activated apoplastic PLCP could in turn induce the expression of SA related immune genes. In this study, the cis-elements in the promoters of $V v P L C P$ genes was predicted and SA responsive element (TCA-element) observed in $11 V v P L C P$ genes, especially in promoter regions of $V v R D 21-1, V v X B C P 1, V v S A G 12-$ 1 , and $V V A L P 1$. These results indicated that the interaction of SA with $P L C P$ plays a key role in the regulation of upstream defense genes under pathogen attack.

Plasmopara viticola has a destructive influence on grapes worldwide. In this study, we studied separately the expression of PLCP and the surface differences between resistant variety Vitis rootstocks 'Kober 5BB' and susceptible variety $V$. vinifera $c v$. 'Zitian Seedless' after inoculation with $P$. viticola. They were selected for a more detailed temporal analysis of the necrotic lesion formation. After P. viticola strains infection, the two Vitis cultivars showed different infection symptoms: HR-like necrotic spots could be observed on 'Kober 5BB', meanwhile downy mildew sporangia could be observed on cv. 'Zitian Seedless'. At the level of gene expression, the $P L C P$ genes in the two species were also very different. In these two grapevine varieties, the prominently expressed $P L C P$ genes are basically belonged to the RD21, CEP, and $S A G$ subfamilies. This is similar to other previous reports [36], such as, SAG12 subfamily exhibits an increased protein accumulation in Candidatus Liberibacter asiaticus infected trees in citrus [38]. Significant expression of members of the $S A G$ subfamily and RD19 subfamily were also found in $V$. vinifera $c v$. 'Zitian Seedless', and there was almost no change in Vitis rootstocks
'Kober 5BB'. According to previous reports, RD19 subfamily is required for RRS1-R (resistant to Ralstonia solanacearum 1-R) mediated resistance against the bacterial type III effector PopP2 (Pseudomonas outer protein P2) in Arabidopsis [39]. This indicated that the RD19 subfamily might be involved in the resistance to downy mildew in V. vinifera $c v$. 'Zitian Seedless'. Therefore, further research is necessary to demonstrate the participation of $P L C P$ genes in the grapevine response to biotic stress.

Previous research demonstrated that some of the plant $P L C P$ genes, such as $X C P 2$ and $C E P 2$, are common targets of pathogen effectors [25]. Given this, the role of PLCPs in response to biotic stresses needs more information in Vitis. Further we are planing to conduct related experiment such as Yeast two-hybrid, grape transgenic over-expression assay for confirming $V v P L C P s$ interacted effectors' possibility, as well as PCD regulating's function during Plasmopara Viticola infection. In this study, we found that some grape PLCPS genes responded to downy mildew treatments by transcriptomics data and qPCR. Especially $V v R D 21-1$ at $72 \mathrm{~h}$ up-regulated on resistant cultivar during infecton, indicating it might display significant characters in the grapevine protection, but further research is needful to demonstrate that it participate in biotic stress responses in grapevine.

\section{Conclusions}

In this study, we identified $23 V v P L C P$ genes in grapevine and performed the systematic and comprehensive analysis of the $V v P L C P$ gene family, including conserved domain, phylogenetic relationship, gene structure, chromosome location, gene duplication, cis-acting elements, and expression pattern analysis. We determined the numerous cis-acting elements in the $V v P L C P$ promoter sequences, indicating that $V v P L C P$ genes participate in the complex regulatory networks regarding development and responses to biotic and abiotic stresses. The transcriptional responses of $V v P L C P$ in different times indicated that $V v P L C P$ could act in a time-dependent behavior in attacked grapes by pathogen. Taken together, genome-wide analysis of the $V \nu P L C P$ will provide a solid foundation for functional analyses of PLCP genes in grapevine, and further study on several PLCP genes help to understand their biological functions.

\section{Materials and methods}

\section{Sequence retrieval for grapevine papain-like cysteine proteases}

For sequence retrieval and identification of PLCP proteins, we used available genome databases for grapevine (http://www.rosaceae.org/projects/grape_genome) and Arabidopsis thaliana (TAIR10: http://www.arabidopsis.org/) [40]. The sequence of other species including, 
Oryza sativa and Glycine max were downloaded from phytozome (https://phytozome.jgi.doe.gov/pz/portal. html). Moreover, initial identification of PLCP was carried out using HMMER (http://www.hmmer.org/) against the Pfam (http://pfam.xfam.org/) Peptidase_C1 domain (PF00112) with default settings. Afterwards, the identified proteins sequences were further verified for PLCP domain compositions in SMART databases (http://smart. embl-heidelberg.de/) [41] and NCBI-Conserved Domain database (https://www.ncbi.nlm.nih.gov/Structure/cdd/ wrpsb.cgi) [42]. By comparing the results of two databases, the protein sequences with errors, shorter length $(<100 \mathrm{bp})$, and absence of Peptidase_C1 domain were eliminated.

\section{Multiple sequence alignment (MSA) and phylogenetic characterization of PLCP}

Full-length amino acid sequences of PLCP were used for initial multiple sequence alignment by MUSCLE method [43] with default parameters. Manual correction was performed to remove poorly aligned regions using BioEdit software [44]. Sequences after screening were used to construct a phylogenetic tree using the maximum likelihood (ML) method in MEGA software with the default options [45]. The circular tree was constructed using the 1000 bootstrap replicated by the Jones, Taylor, and Thornton amino acid substitution model (JTT model), keeping the other parameters set to the defaults to determine the reliability of the resulting tree.

\section{Protein properties, exon-intron structure analysis and conserved motif analysis}

The ExPASY PROTPARAM tool (http://web.expasy .org/ protparam/) [46] was consulted for various physicochemical properties, such as molecular weight $(M W)$, theoretical isoelectronic points ( $\mathrm{pI}$ ), and instability index. The subcellular locations of grapevine PLCP proteins were predicted by WoLF PSORT (http://www.genscript.com/psort/ wolf_psort.html) [45]. The exon/intron/UTR structures of PLCP were analyzed using the gene structure display server program called GSDS2.0 website (http://gsds.cbi. pku.edu.cn/) [47]. The motif logos of the $V v P L C P$ were generated by submitting the sequences to the MEME website (https://meme-suite.org/meme/index.html), and the optimized parameters were as follows: the number of motifs; 15 and the optimum width of each motif; between 6 and 50 residues.

\section{Chromosomal location and Synteny correlation}

All $V v P L C P$ genes were mapped to grapevine chromosomes based on physical positions at the Grape Genome website (http://plants.ensembl.org/Vitis_vinifera/Info/ Index). TBtools software was used for drawing the map.
Tandem duplicated genes were determined by detecting their physical locations on specific chromosomes and were identified as adjacent paralogous on a grapevine chromosome, with no more than one intervening gene. For joint analysis, we downloaded and analyzed the joint blocks in the grapevine genome from the plant genome replication database. For duplicate pairs, Ka (nonsynonymous substitution rate) and Ks (synonymous substitution rate) and evolutionary constraint $(\mathrm{Ka} / \mathrm{Ks})$ between paralogous pairs of $V v P L C P$ genes were calculated by ParaAT and KaKs_Calculator as previous reports [47, 48].

\section{Cis-element analysis for VvPLCP gene promoter}

We selected $2000 \mathrm{bps}$ upstream of VvPLCPs coding regions as promoter sequence and retrieved them from grapevine genome database (https://wwwdev.genoscope. cns.fr/vitis). PlantCARE online program (http://bioin formatics.psb.ugent.be/webtools/plantcare/html/) was used to search for assumed cis-acting elements. Subsequently, the predicted cis-acting elements of the promoter were visualized using TBtools software [48].

\section{Plant material and $P$. viticola inoculum preparation for inoculation}

V. vinifera $c v$. 'Zitian Seedless' and Vitis rootstocks 'Kober 5BB' were cultivated in the Grape Repository of Nanjing Agricultural University, Nanjing, Jiangsu, China under the natural environment with normal management. The leaves (from 4th to the 7th of shoot) related to each grapevine genotype were detached and thoroughly rinsed at both sides with distilled water. The freshly excised leaves were used for the next infection experiment. Single-sporangia strains of $P$. viticola were used to assure the high reproducibility of results. The strains BS-4-MW was obtained following the downy mildew collection method from Botanical Institute of Hohenheim University, and have been described in the previous report [49]. Mature sporangia of BS-4-MW was collected into a $1.5 \mathrm{~mL}$ eppendorf tube from well-infected leaves and stored in $-80^{\circ} \mathrm{C}$ freezer as inoculum. The sporangial suspension would be prepared from one collected tube by adding $1 \mathrm{~mL}$ distilled water. Afterwards, well-washed leaves were placed with their abaxial side contacting sporangial suspension and kept in a phytochamber (Model: PGX-460C-36L, Saifu, China) with high humidity at $21^{\circ} \mathrm{C}$ in darkness for $24 \mathrm{~h}$. Then the leaves were turned, placing on wet tissue paper with their abaxial side up, and further incubated under a photoperiod of $14 \mathrm{~h}$ light $(25 \mu \mathrm{mol} \cdot \mathrm{m}$ $2 \cdot \mathrm{s}-1)$ with full spectrum lamps and $10 \mathrm{~h}$ darkness $(0 \mu \mathrm{mol} \cdot \mathrm{m}-2 \cdot \mathrm{s}-1)$ at $21^{\circ} \mathrm{C}$. After about $7-10$ days, the entire leaf was covered with freshly downy mildew, which was used for leaf discs phenotype assay. 


\section{Phenotype analysis of infected leaf discs with $P$. viticola}

The newly formed sporangia were brushed into a beaker containing sterile water (the capacity of the beaker is $50 \mathrm{~mL}$, containing $5 \mathrm{~mL}$ of sterile water). Then A hemocytometer (model 40,449,001, Fuchs-Rosenthal, Thoma, Germany) was used to calculate the sporangia concentration of the above-mentioned downy mildew under an optical microscope. The concentration was adjusted to about 40,000 sporangia/mL via gradually adding distilled water by pipette. The new suspension liquid can be used for manual inoculation. Five leaf discs from $V$. vinifera cv. 'Zitian Seedless' and Vitis rootstocks 'Kober 5BB' were prepared to observe the phenotype after spraying BS-4-MW suspension liquid as above. Eight-time points after $P$. viticola infection were set $(0 \mathrm{~h}, 12 \mathrm{~h}, 24 \mathrm{~h}, 48 \mathrm{~h}$, $72 \mathrm{~h}, 96 \mathrm{~h}$, and $168 \mathrm{~h}$ ) and the HR phenomenon pictures were further taken by camera (IMX586,Sony, Japan). Three biological replicates were performed.

\section{RNA extraction, cDNA library construction, and Illumina sequencing}

The total RNA was extracted separately with the Trizol (Invitrogen) method following the steps as described in instructions. The genomic DNA was digested using DNase (TAKARA, Dalian, China). The RNA concentration was measured using a NanoDrop 2000 spectrophotometer (Thermo Scientific, Wilmington, DE, U.S.A.), and RNA quality was assessed on a $1 \%$ denatured agarose gel. The purified mRNA and library construction were performed using a RNA library preparation kit (New England Biolabs, Ipswich, MA, U.S.A.). The constructed library quality was determined using an Agilent 2100 bioanalyzer and an ABI StepOnePlus Real-Time PCR system, and the qualified cDNA library was used for RNAseq. All samples ( $V$. vinifera $c v$.'Zitian Seedless'and Vitis rootstocks'Kober 5BB'at 0, 24, and $72 \mathrm{~h}$ after Plasmopara viticola $\mathrm{BS}-4-\mathrm{MW}$ infection. Three biological replicates were performed.) were sequenced on an Illumina Hiseq 2000 platform conducted by Novogene (Beijing, China, http://www.novogene.cn/).

\section{RNA-Seq analysis}

Clean reads were obtained by removing low-quality reads and reads containing adapter and more than $10 \%$ anonymous nucleotides $(\mathrm{N})$ from raw sequence data (raw reads). Subsequent analyses were conducted based on clean data with high quality and the clean reads were compared to the grapevine gene sequence reference data sets using Bowtie2 [50]. The raw data obtained from Illumina sequencing were deposited in the NCBI Sequencing GEO database (accession number GSM5519225; GSM5519226; $\quad$ GSM5519227; GSM5519229; $\quad$ GSM5519230;
GSM5519232; GSM5519233;

GSM5519234; GSM5519235; GSM5519236; GSM5519237; GSM5519238; GSM5519239; GSM5519240; GSM5519241; and GSM5519242). RSEM tools was used to calculate gene and transcript expression levels using FPKM index (fragments per kilobase of transcript per million mapped reads [51] .

\section{GO annotation of VvPLCPs}

The GO annotation of $V v P L C P S$ was conducted using the "Go Term Enrichment Too" in grapevine Genome Database (http://www.rosaceae.org/projects/grape_genome). The grapevine database was searched to download the detailed gene information of these GO terms. Expression data for $V v P L C P$ genes was obtained from transcriptome data (The expression levels of $V$. vinifera $c v$.'Zitian Seedless'and Vitis rootstocks'Kober 5BB'at 0, 24, and $72 \mathrm{~h}$ after $P$. viticola BS-4-MW infection) using the Novogene tool website (https://magic.novogene.com/customer/ main\#/login). Additionally, FKPM-values (Log2) were used to calculate the fold-change in the gene transcriptional levels, and heat maps were illustrated using the RStudio (R program) [52].

\section{RNA extraction and qPCR}

The RNA was extracted from grapevine leaves treated with $P$. viticola in the inoculation time points using the Trizol (Invitrogen) method as described in instructions. The genomic DNA was digested using DNase (TAKARA, Dalian, China). The cDNA was prepared using the Hifair II 1st Strand cDNA Synthesis SuperMix for qPCR (YEASEN, Shanghai, China). Specific forward and reverse primers of all the identified PLCP genes were designed using Primer premier software (Additional file 10 Table S7). melting curve and amplification curve of primers for qPCR was shown in Additional file $11 \mathrm{Fig}$. S4. The qPCR analysis of all PLCP genes using the cDNA was performed following the guidelines as described in previous studies [53], and grapevine gene Actin and $E F-1 \alpha$ were used as a housekeeping gene for qPCR [54]. The cycle thresholds values $(\mathrm{Ct})$ were used to calculate the relative fold-change. In brief, the qPCR amplification reactions were performed on an ABI 7500 Real Time PCR System (Applied Biosystems, USA) using SYBR Green (Vazyme, Nanjing, China) with three replicates. qPCR amplification mixtures $(20 \mu \mathrm{l})$ contained $2 \mu \mathrm{l}$ template cDNA, iTaq Universial SYBR Green Supermix (10 $\mu$ l) (Applied Bio-systems), 0.4 $\mu \mathrm{l}$ forward primer, $0.4 \mu \mathrm{l}$ reverse primer, and $7.2 \mu \mathrm{l}$ nuclease-free $\mathrm{H}_{2} \mathrm{O}$. The amplification parameters were: denaturation at $95^{\circ} \mathrm{Cfor}$ $5 \mathrm{~min}, 40$ cycles of denaturation at $95^{\circ} \mathrm{C}$ for $10 \mathrm{~s}$, annealing at $60^{\circ} \mathrm{C}$ for 30s. Each cDNA sample was used in triplicate for qPCR analysis. The $\mathrm{Ct}$ values of the triplicate 
reactions for each tested gene were averaged, and then, these values were normalized to that of the Actin (GenBank Accession number AB073011) and EF-1 $\alpha$ (GenBank Accession number EC931777) gene. The relative expression level of each gene was calculated via the $2-\Delta \Delta C T$ formula $[55,56]$. The t-test was conducted using the SPSS software (SPSS 17.0, Chicago, IL, USA, ${ }^{*} P<0.05$, **P $P$ 0.01). The Minimum Information for Publication of Quantitative Real-Time PCR Experiments (MIQE) guidelines [57] were followed for performing the qPCR experiments of PLCP genes.

\section{Abbreviations}

PLCP: Plant papain-like cysteine protease; Ks: Synonymous mutation rate; Ka: Non-synonymous mutation rate; HMM: Hidden Markov Model; BLASTP: Basic Local Alignment Search Tool algorithms; MEME: Multiple Em for Motif Elicitation; CDD: Conserved domains; qPCR: Real-Time PCR; RPKM: Reads Per Kilobase per Million mapped reads; HR: Hypersensitive response; PCD: Programmed cell death; NTC: qPCR experiment without template negative control.

\section{Supplementary Information}

The online version contains supplementary material available at https://doi. org/10.1186/s12870-021-03279-w. Additional file 1: Table S1: Detailed information on the results of grape
PLCP gene screening.

Additional file 2: Table S2: Protein properties of PLCP gene family in grapevine.

Additional file 3: Figure S1: Predict the conservative motif of PLCPs by online MEME website.

Additional file 4: Table S3: The Ka/Ks ratios and divergence between paralogous VVPLCP gene pairs.

Additional file 5: Table S4: Promoter Cis-regulatory elements analysis details of grapevine $V V P L C P$ genes.

Additional file 6: Figure S2: Number of each cis-acting element in the promoter region (2.0 kb upstream of the translation start site) of VVPLCP. Based on the functional annotation, the cis-acting elements were classified into three major classes: plant growth and development, phytohormone responsive, or abiotic and biotic stresses-related cis-acting elements genes.

Additional file 7: Figure S3: Histogram of GO function enrichment of PLCP gene. The abscissa represents the description of the 10 most significant pathways enriched by the PLCP gene. The ordinate represents the $p$ value of the significance test of the difference gene. Different colors represent different $\mathrm{GO}$ types.

Additional file 8: Table S5: The detailed information of the GO function enrichment list of PLCP gene. (XLS $5 \mathrm{~kb}$ )

Additional file 9: Table S6: FPKM expression matrix of PLCP gene, FPKM refers to expected number of Fragments Per Kilobase of transcript sequence per Millions base pairs sequenced.

Additional file 10: Table S7: The primers sequences of $V V P L C P$ genes for QPCR.

Additional file 11: Figure S4: Melting curve and amplification curve for QPCR.

\section{Acknowledgements}

We extend our thanks to the reviewers and editors for their careful reading and helpful comments on this manuscript.

\section{Authors' contributions}

Conceived and designed the experiments: PJG, JGF. Analyzed the data: JK, PJG, MQG, ZJL, MWZ, LFSG. RT-qPCR: JK, PJG. Wrote the paper: JK, PJG, ES. Revised the paper: ES, PJG, LFSG, JGF. All authors worked in developing the final manuscript and read and approved it.

\section{Funding}

This work was supported by the Provincial Natural Science Foundation of Jiangsu (BK20200541), the Natural Science Foundation of China (31672131), the Natural Science Foundation of China (31872047) and the Key R\&D projects in Jiangsu Province (BE2018389). As well, this work is funded by the Academic Program Development of Jiangsu Higher Education Institutions.

\section{Availability of data and materials}

All data generated or analyzed during this study are included in this published article (and its Additional files). All data and material used in this study are available from the corresponding author upon reasonable request.

\section{Declarations}

Ethics approval and consent to participate Not applicable.

\section{Consent for publication}

Not applicable.

\section{Competing interests}

The authors declare that they have no competing interests.

Received: 16 March 2021 Accepted: 19 October 2021

Published online: 30 October 2021

\section{References}

1. Diaz-Mendoza M, Velasco-Arroyo B, Gonzalez-Melendi P, Martinez M, Diaz I. C1A cysteine protease-cystatin interactions in leaf senescence. J Exp Bot. 2014;65(14):3825-33.

2. Novinec M, Lenarcic B. Papain-like peptidases: structure, function, and evolution. Biomol Concepts. 2013;4(3):287-308.

3. Rawlings ND, Barrett AJ, Bateman A. MEROPS: the peptidase database. Nucleic Acids Res. 2010;38:D227-D33.

4. Rawlings ND, Barrett AJ, Bateman A. MEROPS: the database of proteolytic enzymes, their substrates and inhibitors. Nucleic Acids Res. 2012;40(D1):D343-D50.

5. Turk $V$, Turk B, Turk D. Lysosomal cysteine proteases: facts and opportunities. EMBO J. 2001;20(17):4629-33.

6. Rehm FBH, Jackson MA, De Geyter E, Yap K, Gilding EK, DurekT, et al. Papain-like cysteine proteases prepare plant cyclic peptide precursors for cyclization. Proc Natl Acad Sci U S A. 2019;116(16):7831-6.

7. Dubey VK, Pande M, Singh BK, Jagannadham MV. Papain-like proteases: Applications of their inhibitors. Afr J Biotechnol. 2007;6:9.

8. Richau KH, Kaschani F, Verdoes M, Pansuriya TC, Niessen S, Stuber K, et al. Subclassification and biochemical analysis of plant papain-like cysteine proteases displays subfamily-specific characteristics. Plant Physiol. 2012;158(4):1583-99.

9. Berti PJ, Storer AC. Alignment/phylogeny of the papain superfamily of cysteine proteases. J Mol Biol. 1995;246(2):273-83.

10. Kordis $D$, Turk V. Phylogenomic analysis of the cystatin superfamily in eukaryotes and prokaryotes. BMC Evol Biol. 2009;9:266.

11. Hierl G, Vothknecht U, Gietl C. Programmed cell death in Ricinus and Arabidopsis: the function of KDEL cysteine peptidases in development. Physiol Plant. 2012;145(1):103-13.

12. Ahmed SU, Rojo E, Kovaleva V, Venkataraman S, Dombrowski JE, Matsuoka $\mathrm{K}$, et al. The plant vacuolar sorting receptor AtELP is involved in transport of $\mathrm{NH} 2$-terminal propeptide-containing vacuolar proteins in Arabidopsis thaliana. J Cell Biol. 2000;149(7):1335-44.

13. Liu H, Hu M, Wang Q, Cheng L, Zhang Z. Role of papain-like cysteine proteases in plant development. Front Plant Sci. 2018;9:1717. 
14. Nino MC, Kang KK, Cho YG. Genome-wide transcriptional response of papain-like cysteine protease-mediated resistance against Xanthomonas oryzae pv. Oryzae in rice. Plant Cell Rep. 2020;39(4):457-72.

15. Martinez M, Cambra I, Carrillo L, Diaz-Mendoza M, Diaz I. Characterization of the entire cystatin gene family in barley and their target Cathepsin L-like cysteine-proteases, Partners in the Hordein Mobilization during seed germination. Plant Physiol. 2009:151(3):1531-45.

16. Lin E, Burns DJW, Gardner RC. Fruit developmental regulation of the kiwifruit actinidin promoter is conserved in transgenic petunia plants. Plant Mol Biol. 1993;23(3):489-99.

17. van Wyk SG, Du Plessis M, Cullis CA, Kunert KJ, Vorster BJ. Cysteine protease and cystatin expression and activity during soybean nodule development and senescence. BMC Plant Biol. 2014;14:294.

18. Guo Y, Gan SS. Translational researches on leaf senescence for enhancing plant productivity and quality. J Exp Bot. 2014;65(14):3901-13.

19. Lohman KN, Gan SS, John MC, Amasino RM. Molecular analysis of natural leaf senescence in Arabidopsis thaliana. Physiol Plant. 1994;92(2):322-8.

20. Konno K, Hirayama C, Nakamura M, Tateishi K, Tamura Y, Hattori M, et al. Papain protects papaya trees from herbivorous insects: role of cysteine proteases in latex. Plant J. 2004;37(3):370-8.

21. Pechan T, Ye L, Chang Y, Mitra A, Lin L, Davis FM, et al. A unique 33-kD cysteine proteinase accumulates in response to larval feeding in maize genotypes resistant to fall armyworm and other Lepidoptera. Plant Cell. 2000;12(7):1031-40.

22. Gessler C, Pertot I, Perazzolli M. Plasmopara viticola: a review of knowledge on downy mildew of grapevine and effective disease management. Phytopathol Mediterr. 2011;50(1):3-44.

23. Chen W-J, Delmotte F, Richard-Cervera S, Douence L, Greif C, Corio-Costet M-F. At least two origins of fungicide resistance in grapevine downy mildew populations. Appl Environ Microbiol. 2007;73(16):5162-72.

24. Jashni MK, Mehrabi R, Collemare J, Mesarich $\mathrm{CH}$, de Wit P. The battle in the apoplast: further insights into the roles of proteases and their inhibitors in plant-pathogen interactions. Front Plant Sci. 2015;6:584.

25. Misas-Villamil JC, van der Hoorn RAL, Doehlemann G. Papain-like cysteine proteases as hubs in plant immunity. New Phytol. 2016;212(4):902-7.

26. Shindo T, Van Der Hoorn RAL. Papain-like cysteine proteases: key players at molecular battlefields employed by both plants and their invaders. Mol Plant Pathol. 2008:9(1):119-25.

27. Pechan T, Cohen A, Williams WP, Luthe DS. Insect feeding mobilizes a unique plant defense protease that disrupts the peritrophic matrix of caterpillars. Proc Natl Acad Sci U S A. 2002;99(20):13319-23.

28. Zhang S, Xu Z, Sun H, Sun L, Shaban M, Yang X, et al. Genome-wide identification of papain-like cysteine proteases in Gossypium hirsutum and functional characterization in response to Verticillium dahliae. Front Plant Sci. 2019;10:134

29. Yuan S, Ke D, Li R, Li X, Wang L, Chen H, et al. Genome-wide survey of soybean papain-like cysteine proteases and their expression analysis in root nodule symbiosis. BMC Plant Biology. 2020;20:1.

30. Cannon SB, Mitra A, Baumgarten A, Young ND, May G. The roles of segmental and tandem gene duplication in the evolution of large gene families in Arabidopsis thaliana. BMC Plant Biol. 2004:4:10.

31. Zou Z, Huang Q, Xie G, Yang L. Genome-wide comparative analysis of papain-like cysteine protease family genes in castor bean and physic nut. Sci Rep. 2018:8:1.

32. Liu J, Sharma A, Niewiara MJ, Singh R, Ming R, Yu Q. Papain-like cysteine proteases in Carica papaya: lineage-specific gene duplication and expansion. BMC Genomics. 2018;19:1.

33. Zhai $Y$, Cui Y, Song M, Vainstein A, Chen S, Ma H. Papain-like cysteine protease gene family in fig (Ficus carica L.): genome-wide analysis and expression patterns. Front. Plant Sci. 2021;12:681801.

34. Zou Z, Xie G, Yang L. Papain-like cysteine protease encoding genes in rubber (Hevea brasiliensis): comparative genomics, phylogenetic, and transcriptional profiling analysis. Planta. 2017;246(5):999-1018.

35. van der Linde K, Hemetsberger C, Kastner C, Kaschani F, van der Hoorn RA, Kumlehn J, et al. A maize cystatin suppresses host immunity by inhibiting apoplastic cysteine proteases. Plant Cell. 2012:24(3):1285-300.

36. Shindo T, Misas-Villamil JC, Horger AC, Song J, van der Hoorn RA. A role in immunity for Arabidopsis cysteine protease RD21, the ortholog of the tomato immune protease C14. PLoS One. 2012;7(1):e29317.
37. Kaschani F, Shabab M, Bozkurt T, Shindo T, Schornack S, Gu C, et al. An effector-targeted protease contributes to defense against Phytophthora infestans and is under diversifying selection in natural hosts. Plant Physiol. 2010;154(4):1794-804.

38. Clark K, Franco JY, Schwizer S, Pang Z, Hawara E, Liebrand TWH, et al. An effector from the Huanglongbing-associated pathogen targets citrus proteases. Nat Commun. 2018;9(1):1718.

39. Bernoux M, Timmers T, Jauneau A, Briere C, de Wit P, Marco Y, et al. RD19, an Arabidopsis cysteine protease required for RRS1-R-mediated resistance, is relocalized to the nucleus by the Ralstonia solanacearum PopP2 effector. Plant Cell. 2008;20(8):2252-64.

40. Lamesch P, Berardini TZ, Li D, Swarbreck D, Wilks C, Sasidharan R, et al. The Arabidopsis information resource (TAIR): improved gene annotation and new tools. Nucleic Acids Res. 2012:40(Database issue):D1202-10.

41. Letunic I, Bork P. 20 years of the SMART protein domain annotation resource. Nucleic Acids Res. 2018:46(D1):D493-D6.

42. Letunic I, Doerks T, Bork P. SMART 7: recent updates to the protein domain annotation resource. Nucleic Acids Res. 2012:40(Database issue):D302-5.

43. Edgar RC. MUSCLE: a multiple sequence alignment method with reduced time and space complexity. BMC Bioinformatics. 2004;5:113.

44. Alzohairy AM. BioEdit: an important software for molecular biology. Gerf Bull Biosci. 2011;2(1):60-1.

45. Sudhir K, Glen S, Koichiro T. MEGA7: Molecular Evolutionary Genetics Analysis Version 7.0 for Bigger Datasets. Mol Biol Evol. 2016;7:1870.

46. Artimo P, Jonnalagedda M, Arnold K, Baratin D, Csardi G, de Castro E, et al. ExPASy: SIB bioinformatics resource portal. Nucleic Acids Res. 2012:40(Web Server issue):W597-603.

47. Wei H, Wang P, Chen J, Li C, Wang Y, Yuan Y, et al. Genome-wide identification and analysis of B-BOX gene family in grapevine reveal its potential functions in berry development. BMC Plant Biol. 2020;20(1):72.

48. Lescot M. PlantCARE, a database of plant cis-acting regulatory elements and a portal to tools for in silico analysis of promoter sequences. Nucleic Acids Res. 2002;30(1):325-7.

49. Thines M, Zipper R, Schäuffele D, Spring O. Characteristics of Pustula tragopogonis (syn. Albugo tragopogonis) newly occurring on cultivated sunflower in Germany. J Phytopathol. 2010;154(2):88-92.

50. Langmead B, Salzberg SL. Fast gapped-read alignment with bowtie 2. Nat Methods. 2012;9(4):357-9.

51. Dewey CN, Li B. RSEM: accurate transcript quantification from RNASeq data with or without a reference genome. BMC Bioinformatics. 2011;12(1):323

52. Han Y, Li X, Cheng L, Liu Y, Wang H, Ke D, et al. Genome-wide analysis of soybean JmjC domain-containing proteins suggests evolutionary conservation following whole-genome duplication. Front Plant Sci. 2016;7:1800.

53. Khan N, Hu CM, Amjad Khan W, Naseri E, Ke H, Huijie D, et al. Evolution and expression divergence of $E 2$ gene family under multiple abiotic and Phytohormones stresses in Brassica rapa. Biomed Res Int. 2018;2018:5206758

54. Monteiro F, Sebastiana M, Pais MS, Fiqueiredo A. Reference gene selection and validation for the early responses to downy mildew infection in susceptible and resistant Vitis vinifera cultivars. PLoS One. 2013;8(9):e72998.

55. Shangguan L, Chen M, Fang X, Xie Z, Gong P, Huang Y, et al. Comparative transcriptome analysis provides insight into regulation pathways and temporal and spatial expression characteristics of grapevine (Vitis vinifera) dormant buds in different nodes. BMC Plant Biol. 2020;20:1.

56. Gong P, Riemann M, Dong D, Stoeffler N, Gross B, Markel A. et al, Two grapevine metacaspase genes mediate ETI-like cell death in grapevine defence against infection of Protoplasma. 2019;25(6):951-69.

57. Bustin SA, Benes V, Garson JA, Hellemans J, Huggett J, Kubista M, et al. The MIQE guidelines: minimum information for publication of quantitative real-time PCR experiments. Clin Chem. 2009;55(4):611-22.

\section{Publisher's Note}

Springer Nature remains neutral with regard to jurisdictional claims in published maps and institutional affiliations. 